\title{
Application and Research of Intelligent Robot
}

\author{
Mingyun Kou \\ WUWEI OCCUPATIONAL COLLEGE, Wuwei 733000, Gansu, China. E-mail: kouminyun@163.com
}

Abstract: Intelligent robot is the product of more and more intelligent robot under the development of science and technology. Its continuous upgrading has greatly promoted the development of modern society and brought great changes to our production and life. This change will continue with the development of intelligent robot technology. It can be predicted that human life will become more and more intelligent in the future. This paper briefly describes the overview and four development stages of intelligent robot, and introduces the application of intelligent robot in military field, commercial field, industrial agriculture field and medical field, and analyzes the research direction of intelligent robot in the future.

Keywords: Intelligent Robot; Application Field; Research Direction

From the simplest robot in the industrial period to today's intelligent robot, human research and development of robots has never stopped. Along the way, robots have experienced four stages of development and each step up means a new era of robots. At present, robots have ushered in the era of full intelligence. Robots have expanded from the previous industrial applications to the military, medical and even commercial applications, and have officially entered the daily life of ordinary consumers. There are more and more types of smart home, which brings people a more and more convenient lifestyle and greatly improves the happiness index of modern life.

\section{Overview and development stage of intelligent robot}

\subsection{Overview of intelligent robot}

On the basis of ordinary robots, intelligent robots increase the ability of perceiving the surrounding environment, analyzing the surrounding environment independently, and making corresponding processing behaviors. It not only has a variety of internal components, but also has a fully automated control device. Compared with ordinary robots, intelligent robots are smarter with three abilities of perception, interaction and motion $^{[1]}$.

\subsection{The development stage of intelligent robot}

The development of intelligent robot has gone through four stages so far. The first stage was the early stage of the industrial period. At that time, the robot was not intelligent. It was only through computer programming that the robot repeatedly operated some simple actions. Its working principle was based on the servo theory and mechanism theory. The emergence of this robot greatly improved the production efficiency of the industrial period and began to be widely used. The robot in the second stage increases the memory storage capacity, which benefits from the rapid development of computer technology and sensor technology. In this stage, the robot can not only repeat actions according to the established procedures, but also have the simplest perception response to the surrounding environment. Compared with the second stage, the robot in the third stage has greatly improved in the degree of automation, which is the result of the development of information

Copyright(C 2020 Mingyun Kou

doi: 10.18686/ahe.v4i10.2896

This is an open-access article distributed under the terms of the Creative Commons Attribution Non-Commercial License (http://creativecommons. org/licenses/by-nc/4.0/), which permits unrestricted non-commercial use, distribution, and reproduction in any medium, provided the original work is properly cited. 
processing technology and sensing technology to a certain extent. In this stage, the robot has the rudiment of intelligent robot, which can even sense the changes of the surrounding environment, and timely change the behavior response according to the changes. The robot in the fourth stage is the intelligent robot at the present stage. With the rapid development of computer and communication technology, intelligent robot has been able to carry out deep learning, self-improvement through deep learning, and even human-computer interaction ${ }^{[2]}$.

\section{Application of intelligent robot}

\subsection{Application of intelligent robot in military field}

The application range of intelligent robot is very wide, such as the application in military field. Although this application is still in the initial exploration stage, it has made very gratifying achievements, such as material transportation, actual combat attack, investigation and exploration and other tasks can be handed over to intelligent robots to complete. Boston Dynamics has successfully developed a robot that looks like a big dog for the U.S. military. This intelligent robot can be competent for the transportation of goods and materials, and does a very good job. It is different from the traditional material transportation robot. The quadruped bionic design effectively breaks through the disadvantages of the traditional robot which needs to move forward with the help of wheels. Even in the very complex and changeable terrain environment, the big dog robot can still complete the material transportation task as if walking on the ground. It not only has the external advantages of quadruped bionics, but also has a very hard core inside. With its own computer and sensor, it can calmly face any sudden situation and changing road conditions, and also can be remotely controlled by the operator ${ }^{[3]}$. The application of intelligent robot in the military field, in addition to the big dog robot for material transportation, is more for military UAV. Its ultra-high mobility and concealment advantages make the military UAV widely used in electronic interference, investigation, attack, nuclear explosion, nuclear test sampling and other tasks. With the development of intelligent robot technology, its application in the military field will be more and more in-depth, and effectively improve the comprehensive military strength of our country.

\subsection{Application of intelligent robot in business field}

The application of intelligent robots in the commercial field can be described as blooming everywhere, such as the intelligent delivery robot during the epidemic period. This contactless meal delivery service greatly reduces the infection probability of manual delivery, and also reduces the meal delivery workload to a certain extent. It can be predicted that after the end of the epidemic, this intelligent meal delivery robot will be gradually applied to more hotel restaurants. Intelligent early education robot, intelligent sweeping robot, intelligent home equipment and driverless car have entered the market and are warmly welcomed by the younger generation of consumers. This is also a typical commercial application of intelligent robot, which brings a new way of life for consumers. The application of intelligent robot in the commercial field cannot be ignored in the new retail field. With the development of intelligent robot and new retail, the application cooperation between them is becoming more and more frequent. The most important applications are automatic ice cream machine, coffee machine, milk tea machine and orange juice machine. Most of them are still in the automatic sales stage, but a few of them can be simply automatic production. In view of the increasingly high cost of human services, with the continuous progress of intelligent robot technology, we firmly believe that intelligent robots will be more and more applied in new commercial retail areas ${ }^{[4]}$.

\subsection{Application of intelligent robot in industry and agriculture}

Since the beginning of the industrial era, the application of robot has been uninterrupted. The application of intelligent robot in the industrial field is mainly manifested in multi joint manipulator and free mechanical device. It can complete sorting, assembly, welding, transportation, detection and other work automatically or semi automatically under the program setting. Compared with manual work, it has the advantages of high efficiency and accuracy. The application of intelligent robot in agricultural field is mainly reflected in seeder, harvester, fertilizer applicator, weedier, etc. These agricultural intelligent machines greatly improve the efficiency of agricultural production, alleviate the plight of large-scale agricultural planting labor shortage, and accelerate the process of agricultural modernization.

\subsection{Application of intelligent robot in medical field}

Intelligent robots bring new research directions to the medical field. There are many kinds of medical intelligent robots, 
including intelligent robot for surgery, intelligent robot for nursing service, intelligent robot for assisting rehabilitation treatment and intelligent robot for medical teaching. They are involved in all aspects of doctors' medical treatment. For example, a typical medical intelligent robot—Da Vinci surgical robot, can complete a complex minimally invasive surgical operation under the control of doctors, and has more advantages than doctors' hands-on operation in terms of operation accuracy and degree of trauma to patients.

\section{Research direction of intelligent robot}

\subsection{More smooth voice communication}

The reason why intelligent robots are intelligent, to a large extent, is that they can communicate like people. In the future research direction of intelligent robots, the function of language communication will be the first problem that researchers need to break through. On the existing basis, the voice communication of intelligent robots will be smoother.

\subsection{More agile action}

Although the current intelligent robot can simply imitate some basic human actions, it is still slow and inflexible. If we want to fully imitate human extremely rich body movements and reach the point of lifelike, it needs further improvement of intelligent robot technology.

\subsection{Stronger energy storage}

At present, all activities of intelligent robot need power support, so the storage capacity of battery determines the continuous activity time of intelligent robot. In view of the current situation that the continuous activity time of robot is limited, how to increase the storage capacity, prolong the activity time and reduce the charging time is one of the development directions of intelligent robot in the future ${ }^{[5]}$.

\subsection{Deeper self-learning}

In order to work more intelligently for human beings, it is necessary to strengthen the self-learning ability of intelligent robots and implant more advanced brain chips into intelligent robots, so that intelligent robots can complete tasks by themselves without human help, and realize real intelligence.

\section{Conclusion}

Although the intelligent robot at this stage has made outstanding performance compared with the previous stage, there is still a large space for us to explore. How to make robots communicate with people better and help them to take part in production activities to the maximum extent is the development direction of intelligent robots in the future. In order to better carry out the application and research of intelligent robot, the cultivation of artificial intelligence professionals is the top priority. Colleges and universities should shoulder this responsibility, train a large number of high-quality artificial intelligence talents for China's intelligent robot industry, and lead China's intelligent robot industry to a new level.

\section{References}

1. Xiao Z. Research on intelligent robot technology and application based on three capabilities. China New Communications 2018; 20(7): 143-144.

2. Dong W. Status and development of intelligent robots. Mechanical Manufacturing 2019; 57(1): 36-38.

3. Zhao F, Yu H, Zhang H, et al. Research on application technology of intelligent robot. Information Recording Materials 2020; 21(5): 189-190.

4. Zhou H, Wang Y, Wang H, et al. Thinking and research on the application of intelligent robots in the new retail field. Dual Use Technologies and Products for Military and Civil 2018; (13): 35-37.

5. Wang L. Discussion on intelligent robot technology and its application under the environment of internet of things. Scientific and Technological Innovation 2020; (8): 68-69. 